\title{
Male circumcision and the incidence and clearance of genital human papillomavirus (HPV) infection in men: the HPV Infection in men (HIM) cohort study
}

Ginesa Albero ${ }^{1,2,3}$, Xavier Castellsagué ${ }^{1,2}$, Hui-Yi Lin ${ }^{4}$, William Fulp ${ }^{4}$, Luisa L Villa ${ }^{5}$, Eduardo Lazcano-Ponce ${ }^{6}$, Mary Papenfuss ${ }^{4}$, Martha Abrahamsen ${ }^{4}$, Jorge Salmerón ${ }^{6,7}$, Manuel Quiterio ${ }^{6}$, Alan G Nyitray ${ }^{8}$, Beibei Lu ${ }^{4}$, F Xavier Bosch ${ }^{1}$ and Anna R Giuliano ${ }^{4^{*}}$

\begin{abstract}
Background: Reported associations of male circumcision (MC) with human papillomavirus (HPV) infection in men have been inconsistent.

Methods: 4,033 healthy men were examined every six months for a median of 17.5 months. In each study visit, exfoliated cell specimens from the coronal sulcus/glans penis, penile shaft, and scrotum were collected and combined into one sample per person for HPV DNA detection. Samples were tested for 37 HPV types. Cox proportional hazards models were used to evaluate the association between $M C$ and the incidence and clearance of HPV infections and specific genotypes.

Results: The overall incidence of new HPV infections did not differ by MC status (for any HPV, adjusted hazard ratio (aHR) 1.08, 95\% confidence interval (Cl) 0.91-1.27). However, incidence was significantly lower among circumcised versus uncircumcised men for HPV types $58(p=0.01), 68(p<0.001), 42(p=0.01), 61(p<0.001), 71(p<0.001), 81$ $(p=0.04)$, and IS39 ( $p=0.01)$, and higher for HPV types $39(p=0.01)$ and $51(p=0.02)$. Despite the lack of an overall association in the risk of HPV clearance by MC (for any HPV, aHR 0.95, 95\% Cl 0.88-1.02), median times to clearance were significantly shorter among circumcised than uncircumcised men for HPV types $33(p=0.02)$ and $64(p=0.04)$, and longer for HPV types 6 ( $p<0.001), 16(p<0.001)$, and 51 ( $p=0.02)$.

Conclusions: MC is not associated with the incidence and clearance of genital HPV detection, except for certain HPV types. The use of a single combined sample from the penis and scrotum for HPV DNA detection likely limited our ability to identify a true effect of $M C$ at the distal penis.
\end{abstract}

Keywords: Male circumcision, Genital, HPV, Incidence, Clearance

\section{Background}

The majority of sexually active men and women will acquire genital human papillomavirus (HPV) infection at some point during their lifetime. In 2008, an estimated 610,000 cancers in men and women were attributed to HPV infection worldwide [1]. The natural history of cervical HPV infection is well characterized; however, little is known about genital HPV infection in men. A recent randomized controlled trial (RCT) of male circumcision (MC) in Uganda (Africa) showed that MC reduced the

\footnotetext{
* Correspondence: Anna.Giuliano@moffitt.org

${ }^{4}$ H. Lee Moffitt Cancer Center and Research Institute, Tampa, FL 33612, USA Full list of author information is available at the end of the article
}

incidence of high-risk HPV (HR-HPV) infections and increased clearance of HR-HPV infections at the coronal sulcus [2]. However, findings regarding the role of $\mathrm{MC}$ in the incidence and clearance of genital HPV infections have not been consistent across studies [3-5].

Our previously published data regarding the prevalence of genital HPV among all men enrolled in the HPV Infection in Men (HIM) Study showed no overall association between MC and genital HPV infections, except for certain HPV types [6]. The purpose of this study was to determine whether MC affects the incidence and clearance of genital HPV infections in a

\section{Ciomed Central}

( 2014 Albero et al.; licensee BioMed Central Ltd. This is an Open Access article distributed under the terms of the Creative Commons Attribution License (http://creativecommons.org/licenses/by/2.0), which permits unrestricted use, distribution, and reproduction in any medium, provided the original work is properly cited. The Creative Commons Public Domain Dedication waiver (http://creativecommons.org/publicdomain/zero/1.0/) applies to the data made available in this article, unless otherwise stated. 
large, multinational cohort study of healthy men in Brazil, Mexico, and the United States (USA).

\section{Methods}

\section{Study population}

From June 2005 through September 2009, healthy men were enrolled in an ongoing longitudinal study of HPV infection in men, the HIM cohort study. Men were recruited from the general population, universities, and organized health-care systems. Details of the study have been previously described [7]. In brief, men were included if they 1) were aged 18-70 years, 2) were residents of southern Florida, USA, Cuernavaca, Mexico, or São Paulo, Brazil, 3) had no prior diagnosis of penile or anal cancer, 4) had no prior diagnosis of genital or anal warts, 5) had no current diagnosis or symptoms of sexually transmitted infections (STIs), 6) had not participated in an HPV vaccine study, 7) reported no previous diagnosis of HIV, 8) had not been imprisoned, homeless, or received drug treatment during the past six months, 9) were willing to commit to 10 scheduled visits every six months, and 10) had no plans to relocate within the next four years. Men who were eligible to participate reviewed and signed a written informed consent form. Before study initiation, the Human Subjects Committees of the University of South Florida, the Centro de Referencia e Tratamento de Doencas Sexualmente Transmissiveis e AIDS, Brazil, and The National Institute of Public Health of Mexico approved the research protocol.

Consenting participants completed a pre-enrollment visit, an enrollment visit scheduled approximately two weeks later, and eight additional visits after enrollment that occurred every six months over a period of four years. At each study visit, participants completed an 88-item computer-assisted self-interview (CASI) which collected information regarding sociodemographic characteristics, tobacco consumption, and sexual behavior. The primary languages spoken by participants from Brazil, Mexico, and the United States were Portuguese, Spanish, and English, respectively, and the CASI was available in these languages. After the interview, men underwent a clinical examination at which time circumcision status was assessed. Participants with full or partial circumcision were considered circumcised.

\section{Penile and scrotal sampling}

Sampling techniques have been described in detail previously [8]. Briefly, exfoliated epithelial cells from the coronal sulcus/glans penis, penile shaft, and scrotum were collected using three different saline pre-wetted Dacron swabs and combined into one sample before DNA extraction. Among uncircumcised men, the foreskin was sampled at the time of collecting the coronal sulcus/ glans penis specimen. All HPV samples were stored at $-80^{\circ} \mathrm{C}$ prior to polymerase chain reaction (PCR) analysis and genotyping. These sampling procedures were standardized across the three countries, with one clinical site per country. Staff at each site collecting specimens and conducting physical exams were either medical doctors or nurse practitioners.

\section{HPV analyses}

The detailed protocol for HPV analysis has been previously described [8]. Briefly, DNA was extracted using the Media Kit (QIAGen, Valencia, CA, USA), according to the manufacturer's instructions. HPV testing was undertaken by use of PCR for amplification of a fragment of the HPV L1 gene. Specimens were tested for the presence of HPV with the PGMY09/11 L1 consensus primer system [9]. HPV genotyping was conducted on all specimens, regardless of the HPV PCR result, using the Linear Array method (Roche Molecular Diagnostics, Alameda, CA, USA) [10] to detect $37 \mathrm{HPV}$ types. The 13 HPV types classified as oncogenic included: 16, 18, $31,33,35,39,45,51,52,56,58,59$ and 68. Nononcogenic HPV types: 6, 11, 26, 40, 42, 53, 54, 55, 61, $62,64,66,67,69,70,71,72,73,81,82,83,84$, IS39, and 89 (CP6108) [11]. Samples were considered valid if they were positive for $\beta$-globin and/or any HPV DNA genotype.

\section{Statistical analysis}

Differences in the distribution of sociodemographic characteristics, tobacco consumption, and sexual behavior characteristics by circumcision status were compared using the Pearson's chi-square test. Differences in the median time to follow-up by MC status were compared using a nonparametric equality of medians test.

The classification of "any HPV" was defined as a positive test result for at least one of the $37 \mathrm{HPV}$ genotypes included in the Linear Array test. HPV detection of single or multiple oncogenic HPV types was classified as "oncogenic HPV". Similarly, HPV detection of single or multiple nononcogenic HPV types was classified as "non-oncogenic HPV".

HPV incidence by circumcision status was estimated according to different classifications of HPV types: any $\mathrm{HPV}$, oncogenic HPV, non-oncogenic HPV, and for each specific HPV type. For each of the above analyses, only participants free of the relevant HPV type at enrollment were included. Men with concomitant incident HPV infections could contribute to several HPV classifications. Time to newly acquired HPV was estimated using the time from the date of study entry to the date of the first detection of HPV DNA, assuming a new infection arose at the date of detection. The exact 95\% confidence intervals (CIs) for incidence estimates were based on the number of events modeled as a Poisson variable for the total person-months. The unit of observation was 
the individual participant, and each man with an incident HPV infection was counted only once during follow-up using only data from the first occurrence. Cumulative incidence of any HPV, HPV 16, oncogenic $\mathrm{HPV}$, and non-oncogenic HPV types was estimated for circumcised and uncircumcised men using the KaplanMeier method.

Cox proportional hazards models were used to assess the association between HPV incidence and circumcision status [12]. Hazards ratios (HRs) and 95\% CIs were used as measures of association for comparison of circumcised versus uncircumcised men. The proportional hazards assumption for the Cox models was tested [13], and no violations were found, except in the nononcogenic HPV model. The non-oncogenic HPV incidence model was stratified according to age to reduce violations of the proportional hazards assumption.

HPV clearance was defined as a participant testing HPV negative at two subsequent consecutive visits following a positive HPV test result, excluding those testing HPV positive for the first time at a participant's final visit. Time to HPV clearance was estimated by using the time at which the participant first tested positive to the date of the first negative test. Median time to clearance was estimated among incident infections using the Kaplan-Meier method for any HPV, oncogenic HPV, non-oncogenic HPV, and for each specific HPV type, according to circumcision status. Analyses were performed for each individual HPV type. Men whose HPV infections did not clear were censored in the analysis.

Cox proportional hazards models with the robust covariance matrix estimator to account for within-subject correlation were used to assess the association between HPV clearance and circumcision status [12]. Men with HPV infections, regardless of baseline HPV status, were included in the models. HRs and 95\% CIs were used as measures of association for the comparison of circumcised versus uncircumcised men. The proportional hazards assumption for the Cox models was tested [13], and HPV clearance models were stratified according to age and country to reduce violations of the proportional hazards assumption.

An HPV infection was considered persistent if a man was HPV DNA-positive at two or more consecutive visits with the same specific HPV type, and an HPV infection was considered transient if a man was positive only once. When a participant missed a study visit, the results from the next visit were used.

The same variable selection procedure was used to evaluate factors associated with incidence and clearance of HPV infections. Factors that had a $\mathrm{p}$-value $<0.10$ were considered covariates. Backwards selection methods, with a significance threshold of 0.05 , were used to identify covariates for inclusion in the final multivariable model.
Candidate variables included education, marital status, smoking status, lifetime number of female sexual partners, lifetime number of male anal sex partners, number of female sexual partners in the past 3-6 months, number of male anal sex partners in the past 3 months, and sixmonth visit compliance status (i.e. whether the elapsed time between follow-up visits was longer than 6.5 months). Country (USA, Brazil, and Mexico) and age (categorical) were included in all models as study design factors. In addition, HPV status at baseline was a candidate variable in the HPV clearance models.

Statistical analyses were conducted using R 2.13.0 (R Development Core Team) and SAS software, version 9.2 (SAS Institute Inc., Cary, NC). Statistical tests were twosided, with a significance threshold of 0.05 .

\section{Results}

Of the 4,074 initial HIM Study participants, 4,033 contributed valid samples for HPV DNA detection and were included in the analyses. Thus, 19 circumcised and 22 uncircumcised men (chi-square p-value $=0.03$ ) contributed inadequate samples, as determined by both lack of $\beta$-globin detection and absence of any HPV genotype. The median duration of follow-up was 17.5 months (interquartile range [IQR], 6.9 - 31.0 months) for this analysis. There were no significant differences in the median duration of follow-up for circumcised (17.9 months) versus uncircumcised men (17.1 months) ( $\mathrm{p}=0.5$ for nonparametric equality of medians test). More than $60 \%$ of men contributed at least 4 visits (IQR, 3-6 visits). The majority of men were uncircumcised (63.6\%) (Table 1). Sociodemographic and behavioral characteristics of men varied by circumcision status. Circumcised men were generally younger (mean age: 31 vs. 33 years) and were residents of the USA (72.7\%). Statistically significant differences by circumcision status were observed for marital status, sexual orientation, lifetime number of female sexual partners, number of female sexual partners in the past 3-6 months, lifetime number of male sexual partners, number of male anal sexual partners in past 3 months, selfreported diagnosis of STIs, and six-month visit compliance status (Table 1).

\section{HPV incidence by MC status}

Kaplan-Meier analyses showed that the overall incidence of any HPV did not differ by circumcision status ( $\mathrm{P}=$ 0.287, log-rank test). Similarly, incidence of HPV 16 , oncogenic HPV, and non-oncogenic HPV did not differ by circumcision status (Figure 1). Estimates for incidence per 100 person-years of any HPV, oncogenic HPV, and non-oncogenic HPV were 50.5, 28.4, and 39.8, respectively, among uncircumcised men, and 45.6, 28.7, and 34.0, respectively, among circumcised men (Table 2). However, the incidence of oncogenic HPV types 39, 51, 
Table 1 Baseline characteristics of participants in the study cohort

\begin{tabular}{|c|c|c|c|c|c|c|}
\hline & & \multicolumn{2}{|c|}{ Uncircumcised N = 2564} & \multicolumn{2}{|c|}{ Circumcised N= 1469} & \multirow[b]{2}{*}{ P-value } \\
\hline & & $\mathrm{N}$ & $\%$ column & $\mathrm{N}$ & $\%$ column & \\
\hline \multirow[t]{5}{*}{ Age (years) } & & & & & & $<0.0001$ \\
\hline & Mean (SD) & & 0.3) & & 2.2) & \\
\hline & $18-30$ & 1125 & 43.9 & 841 & 57.2 & \\
\hline & $31-44$ & 1125 & 43.9 & 421 & 28.7 & \\
\hline & $45-70$ & 314 & 12.2 & 207 & 14.1 & \\
\hline \multirow[t]{4}{*}{ Country of residence } & & & & & & $<0.0001$ \\
\hline & USA & 247 & 9.6 & 1068 & 72.7 & \\
\hline & Brazil & 1200 & 46.8 & 198 & 13.5 & \\
\hline & Mexico & 1117 & 43.6 & 203 & 13.8 & \\
\hline \multirow[t]{5}{*}{ Marital status } & & & & & & $<0.0001$ \\
\hline & Single & 955 & 37.4 & 861 & 58.7 & \\
\hline & Married & 1040 & 40.7 & 332 & 22.6 & \\
\hline & Cohabiting & 374 & 14.6 & 108 & 7.4 & \\
\hline & Divorced/Separated/Widowed & 187 & 7.3 & 165 & 11.3 & \\
\hline \multirow[t]{3}{*}{ Current smoker } & & & & & & 0.054 \\
\hline & No & 1931 & 75.5 & 1147 & 78.2 & \\
\hline & Yes & 628 & 24.5 & 320 & 21.8 & \\
\hline \multirow[t]{5}{*}{ Sexual orientation } & & & & & & $<0.0001$ \\
\hline & MSW & 2107 & 82.5 & 1285 & 87.8 & \\
\hline & MSWM & 162 & 6.3 & 55 & 3.8 & \\
\hline & MSM & 134 & 5.2 & 42 & 2.9 & \\
\hline & No sex & 151 & 5.9 & 82 & 5.6 & \\
\hline
\end{tabular}

Lifetime number of female sexual partners

$6(2047.3)$

0.025

$\begin{array}{rc}\text { Median (SD) } & \\ 0 & 253 \\ 1 & 193 \\ 2-9 & 1044 \\ 10-19 & 423 \\ 20-49 & 355 \\ 50+ & 124\end{array}$

Number of female sexual partners in past 3-6 months

$\begin{array}{rl}\text { Mean (SD) } & \\ 0 & 722 \\ 1 & 948 \\ 2 & 351 \\ 3+ & 329\end{array}$

$1.4(2.3)$

$\begin{array}{lcc}84.1 & 1334 & 90.9 \\ 4.3 & 41 & 2.8 \\ 6.8 & 55 & 3.7 \\ 4.9 & 37 & 2.5\end{array}$


Table 1 Baseline characteristics of participants in the study cohort (Continued)

\begin{tabular}{|c|c|c|c|c|c|}
\hline \multicolumn{3}{|l|}{ Number of male anal sexual partners in past 3 months } & & & \multirow[t]{2}{*}{0.003} \\
\hline Median (SD) & & & & & \\
\hline None & 2360 & 92.9 & 1406 & 95.7 & \\
\hline 1 & 75 & 3.0 & 25 & 1.7 & \\
\hline 2 & 31 & 1.2 & 16 & 1.1 & \\
\hline $3+$ & 73 & 2.9 & 22 & 1.5 & \\
\hline Diagnosis of STIs, ever & & & & & $<0.0001$ \\
\hline No & 2006 & 81.2 & 1246 & 86.8 & \\
\hline Yes & 463 & 18.8 & 190 & 13.2 & \\
\hline HPV status at baseline & & & & & 0.793 \\
\hline Negative & 847 & 33.0 & 492 & 33.5 & \\
\hline Positive & 1717 & 67.0 & 977 & 66.5 & \\
\hline Six-month visit compliance status & & & & & $<0.0001$ \\
\hline No & 1584 & 61.8 & 846 & 57.6 & \\
\hline Yes & 980 & 38.2 & 623 & 42.4 & \\
\hline
\end{tabular}

Pearson's chi-square test was used for statistical comparison between circumcised and uncircumcised men. Some categories do not sum to the total because of missing values. Subjects with unknown values for each characteristic were not included for calculations of $p$-values.

NOTE: SD, standard deviation; MSW, men who have sex with women; MSWM, men who have sex with women and men; MSM, men who have sex with men, STIs, sexually transmitted infections.

58, and 68, and of non-oncogenic HPV types 42, 61, 71, 81 and IS39, did differ between circumcised and uncircumcised participants. Incidence was significantly higher among circumcised men than uncircumcised men for HPV types 39 (HR 1.47, 95\% CI 1.11-1.94) and 51 (HR 1.30, 95\% CI 1.04-1.61). In contrast, incidence was significantly lower among circumcised men than uncircumcised men for HPV types 58 (HR 0.61, 95\% CI 0.430.88), 68 (HR 0.56, 95\% CI 0.40-0.78), 42 (HR 0.59, 95\% CI 0.38-0.90), 61 (HR 0.59, 95\% CI 0.44-0.78), 71 (HR 0.37, 95\% CI 0.21-0.65), 81 (HR 0.70, 95\% CI 0.50-0.98), and IS39 (HR 0.41, 95\% CI 0.20-0.86). The 12-month cumulative incidence of any HPV, oncogenic HPV, and non-oncogenic HPV among uncircumcised men was $38.7 \%, 23.7 \%$, and $32.5 \%$, respectively, compared with $35.2 \%, 23.3 \%$, and $28.9 \%$, respectively, among circumcised men.

In univariate and multivariate analyses, no differences in the risk of HPV incidence by circumcision status were observed (Table 3). A total of 359 circumcised men (48.2\%) had an incident infection, compared with 555 uncircumcised men (47.3\%). For any HPV, no differences in incidence were found by circumcision status in univariate and multivariate analyses (adjusted hazard ratio [aHR] 1.08, 95\% CI 0.91-1.27). Similarly, no differences in incidence were found by circumcision status for oncogenic HPV (aHR 1.11, 95\% CI 0.94-1.31) and nononcogenic HPV (aHR 1.11, 95\% CI 0.94-1.30). The magnitude of the associations remained the same after adjustment for potential confounders, including sociodemographic characteristics and sexual behavior, or when changing the unit of analysis from men to infection (data not shown). Results were consistent when analyses were stratified by country of residence (data not shown).

\section{HPV clearance by MC status}

Kaplan-Meier analyses showed that the median time to clearance of any HPV infection was significantly longer among circumcised men than uncircumcised men $(\mathrm{P}<0.0001$, log-rank test) (Figure 2). Similarly, median time to clearance of oncogenic HPV and non-oncogenic HPV types was significantly shorter among uncircumcised men than circumcised men. Median time to clearance was also significantly shorter among uncircumcised men than circumcised men for HPV types 6 (HR 0.60, 95\% CI 0.440.81), 16 (HR 0.56, 95\% CI 0.42-0.75), and 51 (HR 0.72, 95\% CI 0.54-0.95) (Table 4). Correspondingly, the probability of clearing an HPV infection was significantly lower among circumcised men compared to uncircumcised men. However, median time to clearance was significantly shorter among circumcised men compared to uncircumcised men for HPV 33 (HR 2.54, 95\% CI 1.12-5.73). Although the median time to clearance for HPV 64 was significantly shorter among circumcised versus uncircumcised men, the corresponding HR did not reach statistical significance (HR 6.06, 95\% CI 0.84-43.74).

Univariate and multivariate analyses for clearance are presented in Table 5. HPV clearance was significantly decreased for any HPV infection among circumcised men compared to uncircumcised men in the univariate analysis (HR 0.92, 95\% CI 0.86-0.99). However, after 


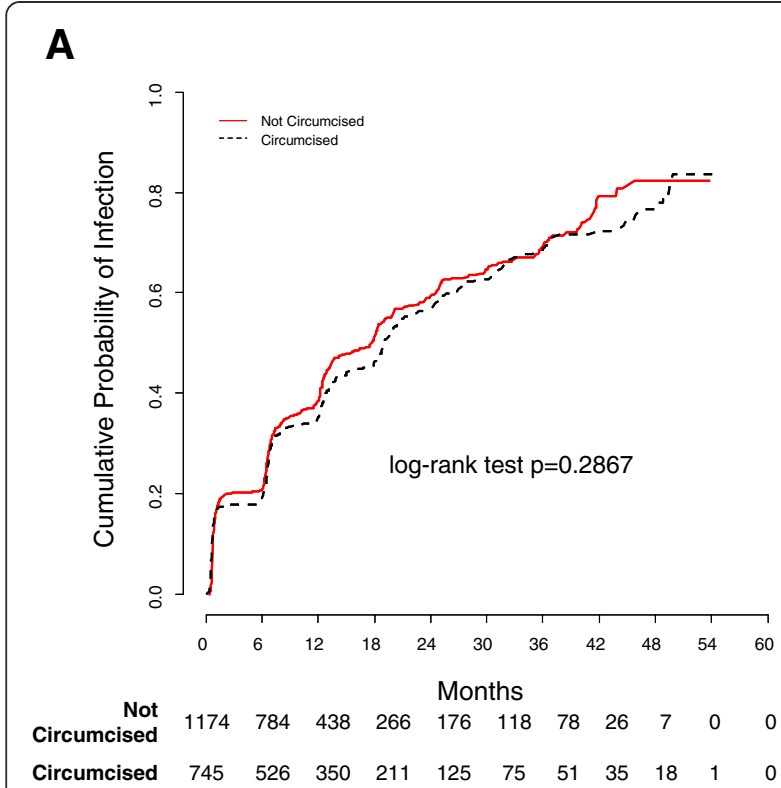

C

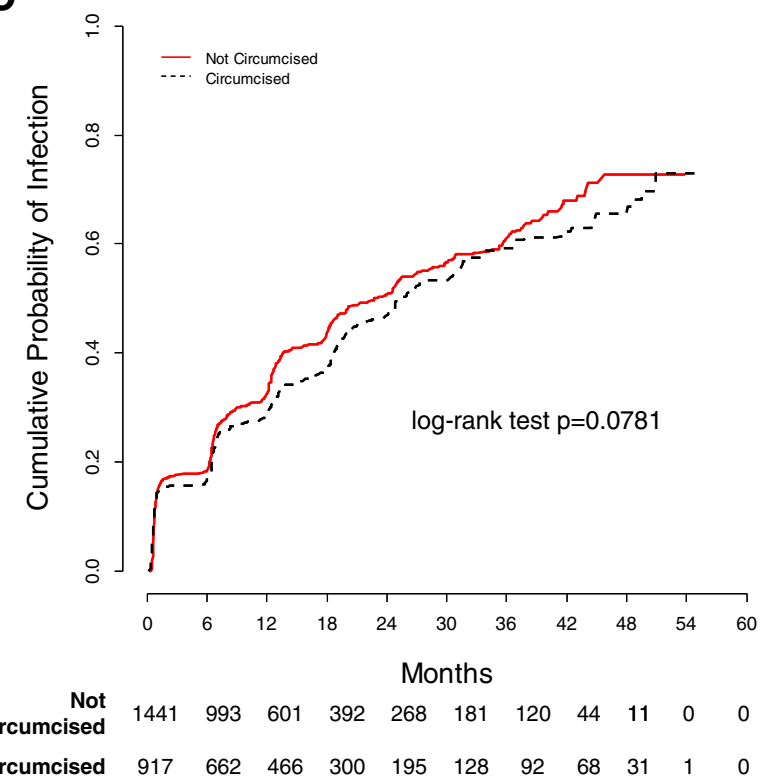

B

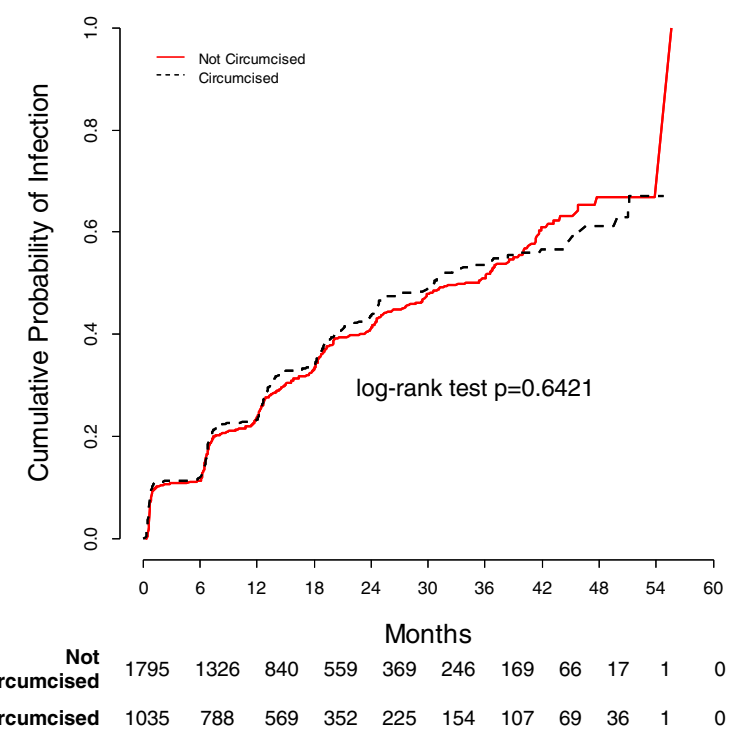

D

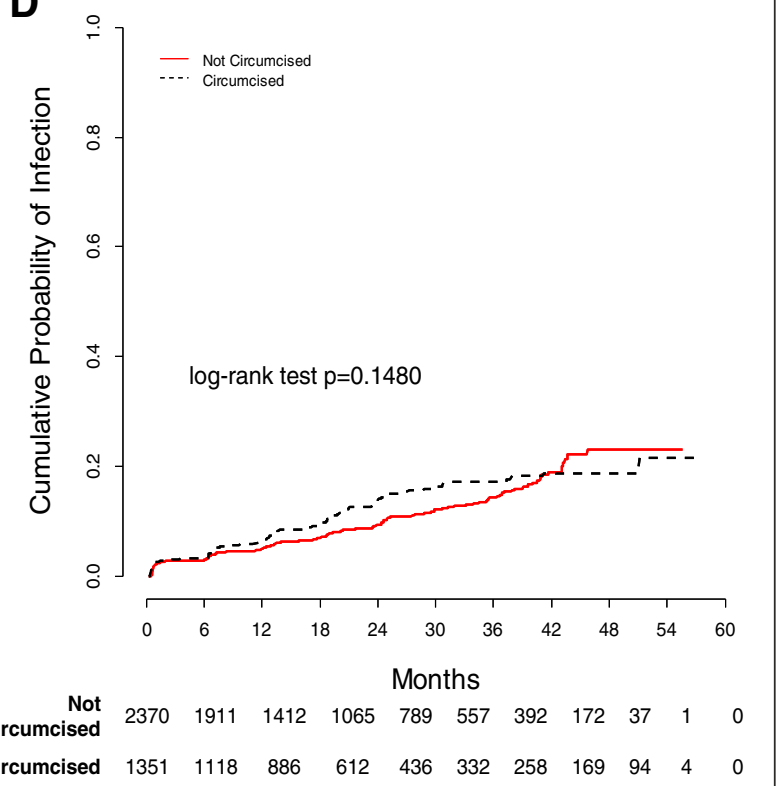

Figure 1 Kaplan-Meier estimates of the cumulative incidence of human papillomavirus (HPV) infections. Kaplan-Meier estimates of the cumulative incidence of human papillomavirus (HPV) infections for any HPV, oncogenic HPV, non-oncogenic HPV, and HPV 16 by male circumcision status: A) Incidence of any HPV; B) Incidence of oncogenic HPV; C) Incidence of non-oncogenic HPV; D) Incidence of HPV 16.

adjustment, this difference was no longer significant (aHR 0.95, 95\% CI 0.88-1.02). Similarly, there were no differences in the risk of clearance for oncogenic HPV (aHR 0.90, 95\% CI 0.81-1.00) or non-oncogenic HPV (aHR 0.98, 95\% CI 0.89-1.07) by circumcision status. The magnitude of the associations with $\mathrm{MC}$ did not change substantially for transient (Table 6) versus persistent (Table 7) HPV infections. Moreover, similar results were observed when analyses were stratified by country of residence (data not shown).

\section{Discussion}

$\mathrm{MC}$ was not associated with an overall reduction in the incidence of genital HPV detection in men. Other longitudinal studies also found no differences in the risk of HPV incidence by MC [3-5]. Lu et al. [3] and Hernandez et al. [4] used combined samples from the coronal sulcus, glans penis, shaft of the penis, and scrotum for HPV detection and found no differences in HPV acquisition by MC. Although VanBuskirk et al. [5] observed site-specific differences in HPV incidence by MC, they 
Table 2 Incidence of HPV by male circumcision status

\begin{tabular}{|c|c|c|c|c|c|c|c|c|}
\hline \multirow[t]{2}{*}{ HPV Type } & \multicolumn{3}{|c|}{ Uncircumcised men } & \multicolumn{3}{|c|}{ Circumcised men } & \multirow{2}{*}{$\begin{array}{c}\text { Log- } \\
\text { rank } \\
\text { test } p\end{array}$} & \multirow{2}{*}{$\begin{array}{c}\text { HR }(95 \% \mathrm{Cl}) \\
\text { [circumcised } \\
\text { versus } \\
\text { uncircumcised] }\end{array}$} \\
\hline & No. Infections/No. PYs & $\begin{array}{c}\text { Incidence per } 100 \\
\text { PYs }(95 \% \mathrm{Cl})\end{array}$ & $\begin{array}{l}\text { \% 12-month } \\
\text { Incidence } \\
(95 \% \mathrm{Cl})\end{array}$ & No. Infections/No. PYs & $\begin{array}{l}\text { Incidence per } 100 \\
\text { PYs }(95 \% \mathrm{Cl})\end{array}$ & $\begin{array}{c}\begin{array}{c}\% \text { 12-month Incidence } \\
(95 \% \mathrm{Cl})\end{array}\end{array}$ & & \\
\hline Any HPV & $555 / 1098$ & $50.5(46.4,54.9)$ & $38.7(35.5,41.7)$ & $359 / 788$ & $45.6(41,50.5)$ & $35.2(31.4,38.8)$ & 0.29 & $0.93(0.81-1.06)$ \\
\hline Oncogenic ${ }^{b}$ & $584 / 2055$ & $28.4(26.2,30.8)$ & $23.7(21.5,25.9)$ & $369 / 1285$ & $28.7(25.9,31.8)$ & $23.3(20.5,26)$ & 0.64 & $1.03(0.84-1.26)$ \\
\hline 16 & $204 / 3546$ & $5.8(5,6.6)$ & $5.1(4.1,6)$ & $143 / 2164$ & $6.6(5.6,7.8)$ & $6.3(4.9,7.7)$ & 0.15 & $1.17(0.95-1.45)$ \\
\hline 18 & $97 / 3886$ & $2.5(2,3)$ & $1.9(1.3,2.5)$ & $71 / 2421$ & $2.9(2.3,3.7)$ & $3.1(2.1,4)$ & 0.24 & $1.20(0.88-1.63)$ \\
\hline 31 & $71 / 3904$ & $1.8(1.4,2.3)$ & $1.6(1.1,2.2)$ & $52 / 2462$ & $2.1(1.6,2.8)$ & $2(1.2,2.8)$ & 0.40 & $1.17(0.81-1.67)$ \\
\hline 33 & $24 / 4039$ & $0.6(0.4,0.9)$ & $0.4(0.1,0.6)$ & $16 / 2532$ & $0.6(0.4,1)$ & $0.8(0.3,1.2)$ & 0.86 & $1.06(0.56-2.00)$ \\
\hline 35 & $44 / 3951$ & $1.1(0.8,1.5)$ & $0.8(0.5,1.2)$ & $26 / 2492$ & $1(0.7,1.5)$ & $1(0.5,1.6)$ & 0.83 & $0.95(0.58-1.55)$ \\
\hline 39 & $106 / 3800$ & $2.8(2.3,3.4)$ & $2.6(1.9,3.3)$ & $95 / 2358$ & $4(3.3,4.9)$ & $3.4(2.3,4.4)$ & 0.01 & $1.47(1.11-1.94)$ \\
\hline 45 & $94 / 3876$ & $2.4(2,3)$ & $1.8(1.2,2.4)$ & $62 / 2451$ & $2.5(1.9,3.2)$ & $2.9(1.9,3.8)$ & 0.70 & $1.06(0.77-1.47)$ \\
\hline 51 & $187 / 3615$ & $5.2(4.5,6)$ & $4.5(3.6,5.5)$ & $142 / 2160$ & $6.6(5.5,7.7)$ & $6.8(5.4,8.3)$ & 0.02 & $1.30(1.04-1.61)$ \\
\hline 52 & $164 / 3525$ & $4.7(4,5.4)$ & $5.4(4.4,6.4)$ & $98 / 2277$ & $4.3(3.5,5.2)$ & $4.3(3.1,5.4)$ & 0.80 & $0.97(0.75-1.24)$ \\
\hline 56 & 79/3894 & $2(1.6,2.5)$ & $2(1.4,2.7)$ & $56 / 2443$ & $2.3(1.7,3)$ & $1.7(0.9,2.4)$ & 0.40 & $1.16(0.82-1.63)$ \\
\hline 58 & $104 / 3845$ & $2.7(2.2,3.3)$ & $2.5(1.8,3.1)$ & $42 / 2467$ & $1.7(1.2,2.3)$ & $1.8(1,2.5)$ & 0.01 & $0.61(0.43-0.88)$ \\
\hline 59 & $165 / 3672$ & $4.5(3.8,5.2)$ & $3.9(3.1,4.8)$ & $119 / 2223$ & $5.4(4.4,6.4)$ & $5(3.7,6.2)$ & 0.14 & $1.19(0.94-1.51)$ \\
\hline 68 & $143 / 3789$ & $3.8(3.2,4.4)$ & $3.9(3,4.7)$ & $48 / 2393$ & $2(1.5,2.7)$ & $2.5(1.6,3.4)$ & $<0.001$ & $0.56(0.40-0.78)$ \\
\hline Non-oncogenic ${ }^{c}$ & $596 / 1497$ & $39.8(36.7,43.1)$ & $32.5(29.7,35.1)$ & $372 / 1094$ & $34(30.6,37.6)$ & $28.9(25.7,32)$ & 0.08 & $0.83(0.69-1.00)$ \\
\hline 6 & $201 / 3553$ & $5.7(4.9,6.5)$ & $5.4(4.4,6.3)$ & $104 / 2258$ & $4.6(3.8,5.6)$ & $3.9(2.8,5)$ & 0.11 & $0.82(0.65-1.04)$ \\
\hline 11 & $58 / 3961$ & $1.5(1.1,1.9)$ & $1.2(0.7,1.7)$ & $25 / 2511$ & $1(0.6,1.5)$ & $1(0.4,1.5)$ & 0.12 & $0.69(0.43-1.10)$ \\
\hline 26 & $21 / 4050$ & $0.5(0.3,0.8)$ & $0.3(0.1,0.6)$ & $6 / 2541$ & $0.2(0.1,0.5)$ & $0.5(0.1,0.8)$ & 0.08 & $0.45(0.18-1.12)$ \\
\hline 40 & $65 / 3941$ & $1.6(1.3,2.1)$ & $1.1(0.6,1.5)$ & $44 / 2479$ & $1.8(1.3,2.4)$ & $1.7(1,2.4)$ & 0.72 & $1.07(0.73-1.58)$ \\
\hline 42 & $78 / 3914$ & $2(1.6,2.5)$ & $1.8(1.2,2.4)$ & 29/2494 & $1.2(0.8,1.7)$ & $0.8(0.3,1.3)$ & 0.01 & $0.59(0.38-0.90)$ \\
\hline 53 & $198 / 3630$ & $5.5(4.7,6.3)$ & $4.6(3.7,5.6)$ & $110 / 2276$ & $4.8(4,5.8)$ & $4.4(3.2,5.5)$ & 0.34 & $0.89(0.71-1.13)$ \\
\hline 54 & $135 / 3815$ & $3.5(3,4.2)$ & $3.8(2.9,4.6)$ & $90 / 2390$ & $3.8(3,4.6)$ & $3.6(2.5,4.6)$ & 0.56 & $1.08(0.83-1.42)$ \\
\hline 55 & $85 / 3873$ & $2.2(1.8,2.7)$ & $1.8(1.2,2.4)$ & $66 / 2434$ & $2.7(2.1,3.4)$ & $2.5(1.6,3.3)$ & 0.16 & $1.26(0.91-1.74)$ \\
\hline 61 & $177 / 3628$ & $4.9(4.2,5.7)$ & $5(4,5.9)$ & $68 / 2437$ & $2.8(2.2,3.5)$ & $3.2(2.2,4.2)$ & $<0.001$ & $0.59(0.44-0.78)$ \\
\hline 62 & 199/3482 & $5.7(4.9,6.6)$ & $5.6(4.6,6.6)$ & $120 / 2231$ & $5.4(4.5,6.4)$ & $5.3(4,6.6)$ & 0.69 & $0.95(0.76-1.20)$ \\
\hline 64 & $11 / 4067$ & $0.3(0.1,0.5)$ & $0.2(0,0.4)$ & $3 / 2557$ & $0.1(0,0.3)$ & $0.1(0,0.2)$ & 0.18 & $0.43(0.12-1.53)$ \\
\hline 66 & $150 / 3708$ & $4(3.4,4.7)$ & $3.8(2.9,4.6)$ & $112 / 2261$ & $5(4.1,6)$ & $5.5(4.2,6.8)$ & 0.08 & $1.25(0.98-1.60)$ \\
\hline 67 & $42 / 4020$ & $1(0.8,1.4)$ & $0.9(0.5,1.3)$ & $22 / 2528$ & $0.9(0.5,1.3)$ & $0.8(0.3,1.3)$ & 0.59 & $0.87(0.52-1.45)$ \\
\hline 69 & 9/4061 & $0.2(0.1,0.4)$ & $0.1(0,0.2)$ & $4 / 2556$ & $0.2(0,0.4)$ & $0.1(0,0.2)$ & 0.60 & $0.73(0.22-2.37)$ \\
\hline
\end{tabular}


Table 2 Incidence of HPV by male circumcision status (Continued)

\begin{tabular}{|c|c|c|c|c|c|c|c|c|}
\hline 70 & $85 / 3872$ & $2.2(1.8,2.7)$ & $2.3(1.6,2.9)$ & $41 / 2467$ & $1.7(1.2,2.3)$ & $1.8(1,2.5)$ & 0.20 & $0.78(0.54-1.14)$ \\
\hline 71 & $64 / 3921$ & $1.6(1.3,2.1)$ & $1.7(1.1,2.3)$ & $15 / 2531$ & $0.6(0.3,1)$ & $0.7(0.2,1.2)$ & $<0.001$ & $0.37(0.21-0.65)$ \\
\hline 72 & $63 / 3928$ & $1.6(1.2,2.1)$ & $1.6(1.1,2.2)$ & $31 / 2503$ & $1.2(0.8,1.8)$ & $1.3(0.7,2)$ & 0.31 & $0.80(0.52-1.23)$ \\
\hline 73 & 70/3923 & $1.8(1.4,2.3)$ & $1.5(1,2)$ & $41 / 2478$ & $1.7(1.2,2.2)$ & $1.6(0.9,2.3)$ & 0.71 & $0.93(0.63-1.37)$ \\
\hline 81 & $113 / 3774$ & $3(2.5,3.6)$ & $3.2(2.4,3.9)$ & 49/2447 & $2(1.5,2.6)$ & $2.1(1.3,2.8)$ & 0.04 & $0.70(0.50-0.98)$ \\
\hline 82 & $43 / 4022$ & $1.1(0.8,1.4)$ & $0.7(0.4,1.1)$ & $34 / 2487$ & $1.4(0.9,1.9)$ & $1.5(0.8,2.1)$ & 0.35 & $1.24(0.79-1.95)$ \\
\hline 83 & $78 / 3864$ & $2(1.6,2.5)$ & $2(1.4,2.6)$ & $45 / 2453$ & $1.8(1.3,2.5)$ & $1.7(1,2.4)$ & 0.60 & $0.91(0.63-1.31)$ \\
\hline 84 & $225 / 3516$ & $6.4(5.6,7.3)$ & $6(4.9,7)$ & $163 / 2125$ & $7.7(6.5,8.9)$ & $7.6(6.1,9.1)$ & 0.09 & $1.19(0.97-1.46)$ \\
\hline 89 & $215 / 3564$ & $6(5.3,6.9)$ & $5.9(4.8,6.9)$ & $140 / 2202$ & $6.4(5.3,7.5)$ & $5.7(4.3,7)$ & 0.51 & $1.07(0.87-1.33)$ \\
\hline IS39 & $35 / 4004$ & $0.9(0.6,1.2)$ & $0.6(0.3,1)$ & $9 / 2543$ & $0.4(0.2,0.7)$ & $0.2(0,0.5)$ & 0.01 & $0.41(0.20-0.86)$ \\
\hline
\end{tabular}

${ }^{2}$ Any HPV was defined as at least one of 37 HPV genotypes;

any HPV was defined as at least one of $37 \mathrm{HPV}$ genotypes;
bOncogenic HPV types: $16,18,31,33,35,39,45,51,52,56,58,59$, and 68 ;

'Non-oncogenic HPV types: $6,11,26,40,42,53,54,55,61,62,64,66,67,69,70,71,72,73,81,82,83,84,89$ (CP6108), and IS39; NOTE: PY, person-year; $\mathrm{HR}$, hazard ratio; $\mathrm{Cl}$, confidence interval. 
Table 3 Univariate and multivariate hazard ratios for the association between male circumcision and incidence of genital HPV infection

\begin{tabular}{|c|c|c|c|c|c|c|c|c|c|c|c|c|}
\hline \multirow[b]{3}{*}{ Circumcision } & \multicolumn{4}{|c|}{ Any HPV } & \multicolumn{4}{|c|}{ Oncogenic HPV } & \multicolumn{4}{|c|}{ Non-Oncogenic HPV } \\
\hline & & & Univariate $^{a}$ & Multivariate $^{\text {b }}$ & & & Univariate $^{a}$ & Multivariate $^{c}$ & & & Univariate $^{a}$ & Multivariate $^{d}$ \\
\hline & Inf./ Total & $\%$ & HR $(95 \% \mathrm{Cl})$ & AHR $(95 \% \mathrm{Cl})$ & Inf./ Total & $\%$ & HR $(95 \% \mathrm{Cl})$ & AHR $(95 \% \mathrm{Cl})$ & Inf./ Total & $\%$ & HR $(95 \% \mathrm{Cl})$ & $\operatorname{AHR}(95 \% \mathrm{Cl})$ \\
\hline No & $555 / 1174$ & 47.3 & 1.00 & 1.00 & $584 / 1795$ & 32.5 & 1.00 & 1.00 & $596 / 1441$ & 41.4 & 1.00 & 1.00 \\
\hline Yes & $359 / 745$ & 48.2 & $1.08(0.92-1.27)$ & $1.08(0.91-1.27)$ & $369 / 1035$ & 35.7 & $1.11(0.94-1.31)$ & $1.11(0.94-1.31)$ & 372/ 917 & 40.6 & $1.09(0.93-1.28)$ & $1.11(0.94-1.30)$ \\
\hline Total & 914/ 1919 & & & & $953 / 2830$ & & & & $968 / 2358$ & & & \\
\hline
\end{tabular}

adjusted for age and country;

bAdjusted for country, age, marital status, lifetime number of female sexual partners, recent number of female sexual partners, recent number of male anal sex partners, and six-month visit compliance status;

‘Adjusted for country, age, marital status, lifetime number of female sexual partners, recent number of female sexual partners, and recent number of male anal sex partners;

${ }^{\mathrm{d} C o x}$ model stratified by age and adjusted for country, marital status, lifetime number of female sexual partners, recent number of female sexual partners, lifetime number of male anal sex partners, and six-month visit compliance status;

NOTE: Unit of analysis: men; Inf., infections; HR, hazard ratio; Cl, confidence interval; AHR, adjusted hazard ratio. 


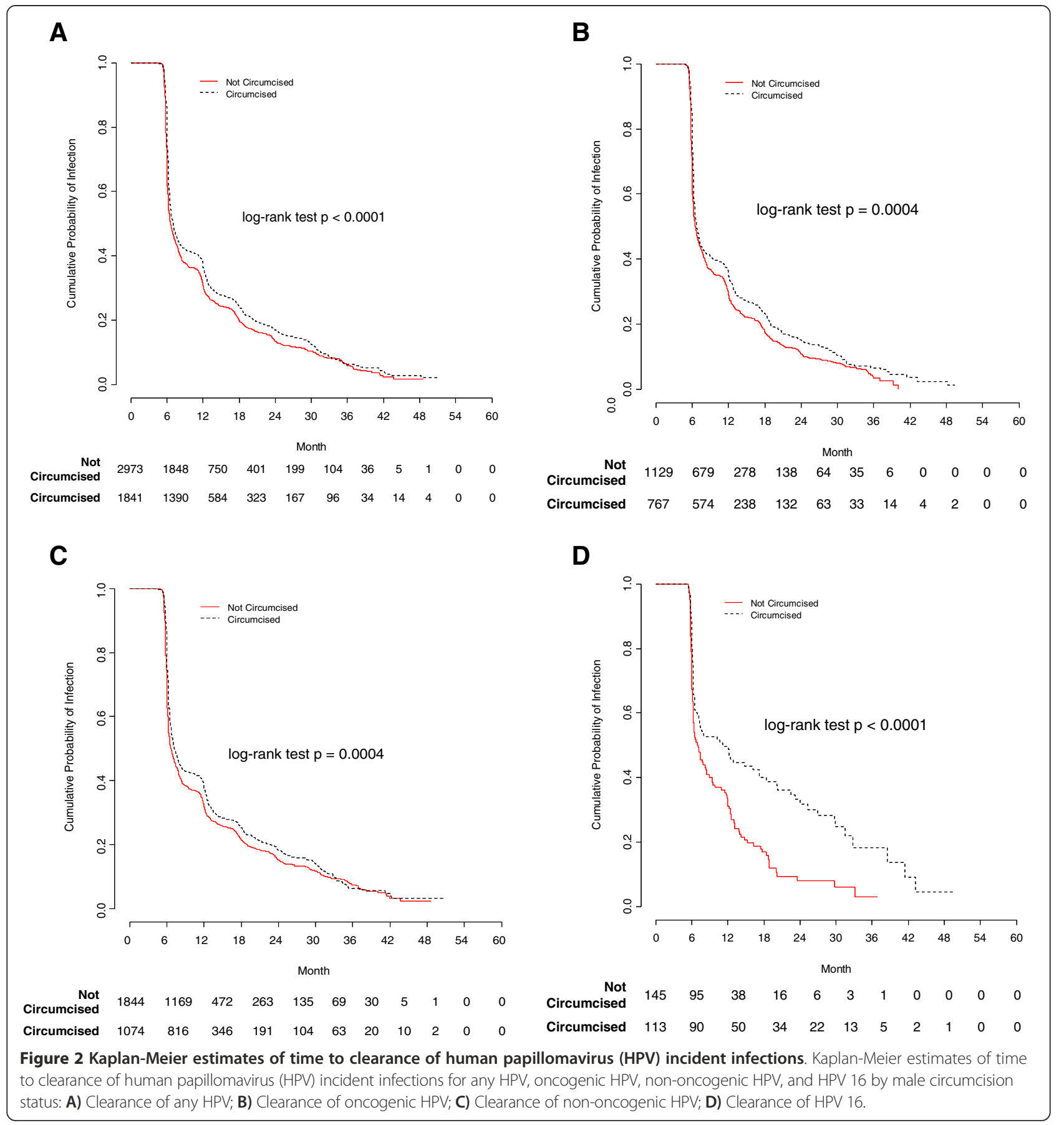

found no differences in overall HPV incidence by MC at any site (penile shaft, glans/corona, and scrotum). Our findings are not consistent with those from a RCT conducted in Uganda, which found that MC reduced the incidence of HR-HPV infections (rate ratio [RR] 0.67, 95\% CI 0.50-0.90) [2]. However, this RCT was not comparable to our study, as they reported associations with specimens collected from only the coronal sulcus [2].

Despite the lack of evidence for an overall association, we did find higher HPV incidences rates in uncircumcised men than in circumcised men for most genotypes. These differences were statistically significant for HPV types 58 , $68,42,61,71,81$, and IS39. In contrast, the incidence of oncogenic HPV types 39 and 51 were significantly higher among circumcised than uncircumcised men. Consistent with our study, VanBuskirk et al. [5] reported higher HPV incidence among uncircumcised than circumcised men for $>50 \%$ of the 21 specific HPV types assessed. Moreover, the RCT in Uganda found that HPV incidence was higher among uncircumcised men 
Table 4 Clearance of HPV by male circumcision status

\begin{tabular}{|c|c|c|c|c|c|c|}
\hline \multirow[t]{2}{*}{ HPV Type } & \multicolumn{2}{|c|}{ Uncircumcised men } & \multicolumn{2}{|c|}{ Circumcised men } & \multirow{2}{*}{$\begin{array}{l}\text { Log- } \\
\text { rank } \\
\text { test } p\end{array}$} & \multirow{2}{*}{$\begin{array}{c}\text { HR }(95 \% \mathrm{Cl}) \\
\text { [circumcised } \\
\text { versus } \\
\text { uncircumcised] }\end{array}$} \\
\hline & $\begin{array}{c}\text { New infections/Cleared } \\
\text { infections }\end{array}$ & $\begin{array}{l}\text { Median time to clearance (months; } \\
\qquad 95 \% \mathrm{Cl} \text { ) }\end{array}$ & $\begin{array}{l}\text { New infections/Cleared } \\
\text { infections }\end{array}$ & $\begin{array}{l}\text { Median time to clearance (months; } \\
\qquad 95 \% \mathrm{Cl} \text { ) }\end{array}$ & & \\
\hline Any HPV & $2973 / 2392$ & $6.6(6.4,6.8)$ & $1841 / 1480$ & $7.1(6.8,7.3)$ & $<0.001$ & $0.85(0.80-0.91)$ \\
\hline Oncogenic ${ }^{\mathbf{b}}$ & $1129 / 937$ & $6.5(6.3,6.8)$ & $767 / 629$ & $6.7(6.4,7.1)$ & $<0.001$ & $0.83(0.75-0.92)$ \\
\hline 16 & $145 / 118$ & $7.1(6.3,8.9)$ & $113 / 79$ & $11.1(7.2,18.4)$ & $<0.001$ & $0.56(0.42-0.75)$ \\
\hline 18 & $71 / 63$ & $6.5(6.1,8.1)$ & $51 / 44$ & $6.3(6.2,7.2)$ & 0.84 & $1.04(0.71-1.54)$ \\
\hline 31 & $49 / 39$ & $7.2(6.5,14.5)$ & $37 / 31$ & $6.4(6.1,6.9)$ & 0.23 & $1.34(0.83-2.17)$ \\
\hline 33 & $16 / 13$ & $6.9(5.9,17.6)$ & $16 / 16$ & $6(5.8,6.6)$ & 0.02 & $2.54(1.12-5.73)$ \\
\hline 35 & $34 / 26$ & $11.3(6.5,18.1)$ & $18 / 15$ & 10.8 (7.1, N.E.) & 0.86 & $0.95(0.50-1.80)$ \\
\hline 39 & $83 / 66$ & $7(6.3,17.3)$ & $71 / 56$ & $11.2(7.4,12.8)$ & 0.68 & $0.93(0.65-1.32)$ \\
\hline 45 & $77 / 65$ & $6.3(6.1,10.6)$ & $51 / 48$ & $6.1(6.1,6.5)$ & 0.23 & $1.26(0.87-1.84)$ \\
\hline 51 & $144 / 116$ & $6.4(6,7.8)$ & $117 / 88$ & $8(6.4,13.1)$ & 0.02 & $0.72(0.54-0.95)$ \\
\hline 52 & $139 / 119$ & $6.4(6,7.6)$ & $84 / 70$ & $6.7(6.4,12)$ & 0.20 & $0.82(0.61-1.11)$ \\
\hline 56 & $58 / 45$ & $6(6,8.9)$ & $44 / 41$ & $6.3(6,7.9)$ & 0.36 & $1.21(0.79-1.86)$ \\
\hline 58 & $72 / 59$ & $6.9(6,11.4)$ & $34 / 26$ & $7.1(6.2,12.9)$ & 0.20 & $0.73(0.46-1.17)$ \\
\hline 59 & $122 / 103$ & $6.2(6,6.7)$ & $89 / 73$ & $6.4(6.2,8.5)$ & 0.11 & $0.78(0.58-1.06)$ \\
\hline 68 & $119 / 105$ & $6.2(6,6.8)$ & $42 / 42$ & $6.4(6.2,10.4)$ & 0.91 & $0.97(0.68-1.39)$ \\
\hline $\begin{array}{l}\text { Non- } \\
\text { oncogenic }\end{array}$ & $1844 / 1455$ & $6.7(6.4,6.9)$ & $1074 / 851$ & $7.2(6.9,7.8)$ & $<0.001$ & $0.86(0.79-0.93)$ \\
\hline 6 & $156 / 142$ & $6.1(6,6.4)$ & $80 / 61$ & $7.8(6.5,12.5)$ & $<0.001$ & $0.60(0.44-0.81)$ \\
\hline 11 & $37 / 27$ & $7.8(6.2,14)$ & $19 / 15$ & $6.6(6,18.1)$ & 0.54 & $1.22(0.65-2.31)$ \\
\hline 26 & $17 / 14$ & $6(5.9,7.1)$ & $5 / 5$ & 6.1 (6, N.E.) & 0.62 & $0.76(0.27-2.13)$ \\
\hline 40 & $47 / 41$ & $6.4(6,7.8)$ & $34 / 27$ & $6.5(6.2,17.6)$ & 0.09 & $0.66(0.40-1.08)$ \\
\hline 42 & $63 / 53$ & $9.1(6.4,12.3)$ & $21 / 18$ & $7.8(6.2$, N.E. $)$ & 0.96 & $1.02(0.59-1.75)$ \\
\hline 53 & $150 / 123$ & $6.7(6.3,7.8)$ & $88 / 68$ & $7.5(6.2,12.5)$ & 0.10 & $0.78(0.58-1.05)$ \\
\hline 54 & $111 / 86$ & $8.3(6.4,12.1)$ & $70 / 56$ & $6.9(6.4,13.1)$ & 0.93 & $1.02(0.72-1.42)$ \\
\hline 55 & $67 / 53$ & $7.2(6.2,12)$ & $50 / 37$ & $7(6.6,18.2)$ & 0.20 & $0.76(0.50-1.16)$ \\
\hline 61 & $126 / 89$ & $6.5(6.1,8.5)$ & $48 / 43$ & $6.9(6.4,13.3)$ & 0.86 & $0.97(0.67-1.40)$ \\
\hline 62 & $148 / 104$ & $7.9(6.5,11.8)$ & $94 / 73$ & $7.9(6.7,12.3)$ & 0.88 & $1.02(0.76-1.38)$ \\
\hline 64 & $8 / 8$ & $6(5.9$, N.E. $)$ & $2 / 2$ & 5.7 (5.5, N.E.) & 0.04 & $6.06(0.84-43.74)$ \\
\hline 66 & $110 / 90$ & $6.8(6.2,8.5)$ & $87 / 69$ & $7.1(6.5,11.9)$ & 0.49 & $0.89(0.65-1.23)$ \\
\hline 67 & $35 / 32$ & $6(6,7.2)$ & $19 / 19$ & $6.3(6,7.4)$ & 0.38 & $1.28(0.71-2.31)$ \\
\hline 69 & $9 / 9$ & $6(5.7$, N.E. $)$ & $2 / 2$ & 7.1 (6.2, N.E.) & 0.99 & $0.98(0.20-4.93)$ \\
\hline
\end{tabular}


Table 4 Clearance of HPV by male circumcision status (Continued)

\begin{tabular}{|c|c|c|c|c|c|c|}
\hline 70 & $62 / 53$ & $6.8(6.2,11.7)$ & $32 / 28$ & $6.7(6.2,12)$ & 0.54 & $1.15(0.73-1.83)$ \\
\hline 71 & $54 / 39$ & $7.5(6.2,13)$ & $14 / 9$ & 7.5 (6, N.E.) & 0.53 & $0.79(0.38-1.64)$ \\
\hline 72 & $49 / 43$ & $6.1(6,7.4)$ & $25 / 23$ & $6.2(6,7.1)$ & 0.35 & $1.28(0.76-2.17)$ \\
\hline 73 & $49 / 39$ & $6.2(6,9.2)$ & $35 / 30$ & $7.6(6.6,13.6)$ & 0.45 & $0.83(0.51-1.33)$ \\
\hline 81 & $94 / 72$ & $7.2(6.4,10.3)$ & $39 / 35$ & $6.6(6.2,11.5)$ & 0.29 & $1.25(0.83-1.88)$ \\
\hline 82 & $30 / 26$ & $6.2(6,11.7)$ & $25 / 21$ & $6.4(6.2,14.2)$ & 0.28 & $0.73(0.40-1.32)$ \\
\hline 83 & $55 / 45$ & $6.9(6,9.5)$ & $32 / 25$ & $8(6.4,13.9)$ & 0.25 & $0.75(0.46-1.22)$ \\
\hline 84 & $170 / 126$ & $7.2(6.4,11.4)$ & $131 / 91$ & $10.4(6.7,13.8)$ & 0.07 & $0.78(0.60-1.03)$ \\
\hline 89 & $166 / 116$ & $7.2(6.3,11.5)$ & $115 / 88$ & $8.4(7.7,12.5)$ & 0.15 & $0.81(0.62-1.07)$ \\
\hline IS39 & $31 / 25$ & $6.2(6,13.2)$ & $7 / 6$ & 13.1 (5.9, N.E.) & 0.78 & $0.87(0.35-2.12)$ \\
\hline
\end{tabular}

${ }^{2}$ Any HPV was defined as at least one of $37 \mathrm{HPV}$ genotypes;

any HPV was defined as at least one of 37 HPV genotypes;
bOncogenic HPV types: $16,18,31,33,35,39,45,51,52,56,58,59$, and 68 ;

'Non-oncogenic HPV types: 6, 11, 26, 40, 42, 53, 54, 55, 61, 62, 64, 66, 67, 69, 70, 71, 72, 73, 81, 82, 83, 84, 89 (CP6108), and IS39;

NOTE: Analysis of clearance was limited to incident HPV detection; NE, not estimable; HR, hazard ratio; $\mathrm{Cl}$, confidence interval. 
Table 5 Univariate and multivariate hazard ratios for the association between male circumcision and clearance of genital HPV infection

\begin{tabular}{|c|c|c|c|c|c|c|c|c|c|c|c|c|}
\hline \multirow[b]{3}{*}{ Circumcision } & \multicolumn{4}{|c|}{ Any HPV } & \multicolumn{4}{|c|}{ Oncogenic HPV } & \multicolumn{4}{|c|}{ Non-Oncogenic HPV } \\
\hline & & & Univariate $^{\mathrm{a}}$ & Multivariate $^{\text {b }}$ & & & Univariate $^{a}$ & Multivariate $^{c}$ & & & Univariate $^{\mathrm{a}}$ & Multivariate $^{d}$ \\
\hline & Inf./ Total & $\%$ & HR $(95 \% \mathrm{Cl})$ & AHR $(95 \% \mathrm{Cl})$ & Inf./ Total & $\%$ & HR $(95 \% \mathrm{Cl})$ & AHR $(95 \% \mathrm{Cl})$ & Inf./ Total & $\%$ & HR $(95 \% \mathrm{Cl})$ & AHR $(95 \% \mathrm{Cl})$ \\
\hline No & $4353 / 5981$ & 72.8 & 1.00 & 1.00 & $1748 / 2266$ & 77.1 & 1.00 & 1.00 & $2605 / 3715$ & 70.1 & 1.00 & 1.00 \\
\hline Yes & $2548 / 3398$ & 75.0 & $0.92(0.86-0.99)$ & $0.95(0.88-1.02)$ & $1089 / 1410$ & 77.2 & $0.86(0.78-0.96)$ & $0.90(0.81-1.00)$ & $1459 / 1988$ & 73.4 & $0.97(0.89-1.05)$ & $0.98(0.89-1.07)$ \\
\hline Total & $6901 / 9379$ & & & & $2837 / 3676$ & & & & $4064 / 5703$ & & & \\
\hline
\end{tabular}

adjusted for age and country;

${ }^{\mathrm{b} C}$ Cox model stratified by country and age, and adjusted for lifetime number of female sexual partners, recent number of male anal sex partners, smoking status, HPV status at baseline, and six-month visit compliance status:

cCox model stratified by country and age, and adjusted for lifetime number of female sexual partners, lifetime number of male anal sex partners, HPV status at baseline, and six-month visit compliance status;

Cox model stratified by country and age, and adjusted for lifetimen nut

${ }^{\mathrm{d}}$ Cox model stratified by

NOTE: Unit of analysis: infections; Abbreviations: Inf., infections; HR, hazard ratio; Cl, confidence interval; AHR, adjusted hazard ratio. 
Table 6 Univariate and multivariate hazard ratios for the association between male circumcision and clearance of transient genital HPV infection

\begin{tabular}{|c|c|c|c|c|c|c|c|c|c|c|c|c|}
\hline \multirow[b]{3}{*}{ Circumcision } & \multicolumn{4}{|c|}{ Any HPV } & \multicolumn{4}{|c|}{ Oncogenic HPV } & \multicolumn{4}{|c|}{ Non-Oncogenic HPV } \\
\hline & & & Univariate $^{\mathrm{a}}$ & Multivariate $^{\mathrm{b}}$ & & & Univariate $^{a}$ & Multivariate $^{c}$ & & & Univariate $^{a}$ & Multivariate $^{d}$ \\
\hline & Inf./ Total & $\%$ & HR $(95 \% \mathrm{Cl})$ & AHR $(95 \% \mathrm{Cl})$ & Inf./ Total & $\%$ & HR $(95 \% \mathrm{Cl})$ & AHR $(95 \% \mathrm{Cl})$ & Inf./ Total & $\%$ & HR $(95 \% \mathrm{Cl})$ & AHR $(95 \% \mathrm{Cl})$ \\
\hline No & $2806 / 4434$ & 63.3 & 1.00 & 1.00 & $1106 / 1624$ & 68.1 & 1.00 & 1.00 & $1700 / 2810$ & 60.5 & 1.00 & 1.00 \\
\hline Yes & $1665 / 2515$ & 66.2 & $0.95(0.88-1.03)$ & $0.98(0.90-1.06)$ & $745 / 1066$ & 69.9 & $0.88(0.77-1.00)$ & $0.91(0.80-1.04)$ & $920 / 1449$ & 63.5 & $0.99(0.89-1.10)$ & $1.01(0.91-1.13)$ \\
\hline Total & $4471 / 6949$ & & & & $1851 / 2690$ & & & & $2620 / 4259$ & & & \\
\hline
\end{tabular}

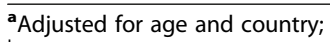

${ }^{\mathrm{b} C o x}$ model stratified by country and age, and adjusted for lifetime number of female sexual partners, recent number of male anal sex partners, smoking status, HPV status at baseline, and six-month visit compliance status;

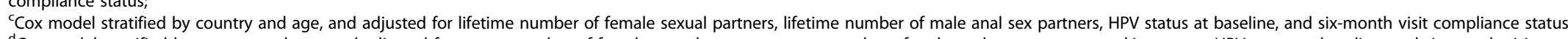
${ }^{d}$ Cox model stratified by country and age, and adjusted for recent number of female sexual partners, recent number of male anal sex partners, smoking status, HPV status at baseline, and six-month visit compliance status.

NOTE: Unit of analysis: infections; Excluded 120 infections that were a combination of persistent and transient infection; Abbreviations: Inf., infections; HR, hazard ratio; Cl, confidence interval; AHR, adjusted hazard ratio. 
Table 7 Univariate and multivariate hazard ratios for the association between male circumcision and clearance of persistent genital HPV infection

\begin{tabular}{|c|c|c|c|c|c|c|c|c|c|c|c|c|}
\hline \multirow[b]{3}{*}{ Circumcision } & \multicolumn{4}{|c|}{ Any HPV } & \multicolumn{4}{|c|}{ Oncogenic HPV } & \multicolumn{4}{|c|}{ Non-Oncogenic HPV } \\
\hline & & & Univariate $^{\mathrm{a}}$ & Multivariate $^{\mathbf{b}}$ & & & Univariate $^{a}$ & Multivariate $^{c}$ & & & Univariate $^{a}$ & Multivariate $^{d}$ \\
\hline & Inf./ Total & $\%$ & HR $(95 \% \mathrm{Cl})$ & AHR $(95 \% \mathrm{Cl})$ & Inf./ Total & $\%$ & HR $(95 \% \mathrm{Cl})$ & AHR $(95 \% \mathrm{Cl})$ & Inf./ Total & $\%$ & HR $(95 \% \mathrm{Cl})$ & AHR $(95 \% \mathrm{Cl})$ \\
\hline No & $1474 / 3102$ & 47.5 & 1.00 & 1.00 & $610 / 1128$ & 54.1 & 1.00 & 1.00 & $864 / 1974$ & 43.8 & 1.00 & 1.00 \\
\hline Yes & $836 / 1686$ & 49.6 & $0.94(0.84-1.05)$ & $0.99(0.88-1.12)$ & $324 / 645$ & 50.2 & $0.76(0.64-0.91)$ & $0.84(0.70-1.01)$ & $512 / 1041$ & 49.2 & $1.07(0.92-1.25)$ & $1.10(0.94-1.29)$ \\
\hline Total & $2310 / 4788$ & & & & $934 / 1773$ & & & & $1376 / 3015$ & & & \\
\hline
\end{tabular}

${ }^{\mathrm{a} A d j u s t e d ~ f o r ~ a g e ~ a n d ~ c o u n t r y ; ~}$

${ }^{\mathrm{b} C o x}$ model stratified by country and age, and adjusted for lifetime number of female sexual partners, recent number of male anal sex partners, smoking status, HPV status at baseline, and six-month visit compliance status;

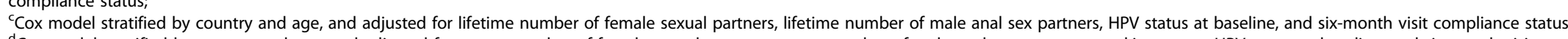
${ }^{\mathrm{d} C o x}$ model stratified by country and age, and adjusted for recent number of female sexual partners, recent number of male anal sex partners, smoking status, HPV status at baseline, and six-month visit compliance status.

NOTE: Unit of analysis: infections; Excluded 120 infections that were a combination of persistent and transient infection; Abbreviations: Inf., infections; HR, hazard ratio; Cl, confidence interval; AHR, adjusted hazard ratio. 
than among circumcised men for all HR-HPV genotypes, being statistically significant for HR-HPV types 18 and 33 [2].

No differences in overall HPV clearance by MC were observed in our study. This finding is in agreement with findings from other longitudinal studies [4,5]. VanBuskirk et al. [5] found that MC had no effect on the likelihood of detecting a persistent versus transient HPV infection over an 8-month period. Hernandez et al. [4] found that the duration of HPV infection did not vary by $\mathrm{MC}$ for the penile shaft, scrotum, or for all genital sites combined. However, the median duration of HPV infection at the glans/coronal sulcus was significantly longer in uncircumcised men that in circumcised men [4]. Similarly, MC increased clearance rates of HRHPV infections at the coronal sulcus in the Uganda RCT [2]. In the study by Lu et al. [3], circumcised men were more likely to clear infection with any HPV and oncogenic HPV types using a combined HPV sample from the coronal sulcus, glans penis, shaft, and scrotum for HPV detection.

Differences in HPV clearance by MC for certain HPV types were observed in the present study. Median times to clearance for HPV types 33 and 64 were significantly shorter among circumcised men compared to uncircumcised men. The RCT in Uganda reported higher rates of clearance among circumcised men than uncircumcised men at the coronal sulcus for most HR-HPV types, but the differences were only statistically significant for HRHPV types 39, 51, and 58 [2]. In contrast, we found that the median times to clearance for HPV types 16,51 , and 6 were significantly longer among circumcised men than uncircumcised men.

Even though our study was not designed to assess the effects of MC and HPV by anatomic genital subsite, there is evidence from the literature that the association between MC and HPV DNA detection varies according to the subsite sampled. Four out of five studies show that the effect of MC in reducing HPV infection is stronger at the glans/corona than at other, more distant, subsites [14-18]. Weaver et al. [14], found that HPV prevalence was significantly lower among circumcised men (17\%) compared to uncircumcised men (32\%) on the glans and foreskin. Interestingly, and consistent with our findings, when combining results from all subsites, overall HPV prevalence was similar between circumcised (31\%) and uncircumcised (29\%) men. Consistent with these results, a study by Nielson et al. [15] reported that HPV prevalence at the glans penis/coronal sulcus was significantly lower among circumcised men (29.8\%) compared to uncircumcised men (35.2\%). However, HPV prevalence combining the glans, shaft, and scrotum was similar in circumcised (48.3\%) and uncircumcised men (44.8\%). In another US study, Hernandez et al. [16] reported a significantly lower HPV prevalence at the glans penis/coronal sulcus among circumcised men (29\%) compared to uncircumcised men (46\%). However, HPV prevalence at the external genitalia was similar among circumcised (63\%) versus uncircumcised (71\%) men. At year 1 in the RCT in Uganda [17], HR-HPV types were more frequently detected on the coronal sulcus than on the penile shaft among both circumcised and uncircumcised men. In addition, HR-HPV prevalence at year 1 on the penile coronal sulcus was significantly lower among circumcised men $(21.5 \%)$ as compared to uncircumcised men (36.3\%). Finally, a study by Partridge et al. [18], conducted among male university students in the USA, found that circumcision status was not associated with incident HPV infection for any of the individual subsites or for all sites combined.

Further supporting the evidence for an effect of MC on HPV infection on the proximal region of the penis, three additional studies reported specific inverse associations between MC and HPV infection in the glans, coronal sulcus, and the distal urethra [19], the glans and coronal sulcus [20], or the urethra [21].

In addition to the subsites included for HPV DNA detection, several other factors may explain the contrasting effects observed across studies, such as differences in participants' ages, the proportion of circumcised men, the number of HPV genotypes included in testing, the time intervals between visits, and the age at which circumcision was performed.

To our knowledge, this is the first international study reporting HPV incidence and clearance by specific HPV types in circumcised and uncircumcised men. With a sample size of 4,033 men, it is also the largest study exploring these associations. A key strength of the present study is its longitudinal design, which allowed repeated measures of genital HPV DNA status for each participant, with a median follow-up of 17.5 months and scheduled visits every six months.

Our study, despite being large, multinational, and prospective, is not free of some limitations, including: (1) the potential for misclassification of HPV infection, as we combined samples from different anatomic subsites; (2) the potential for misclassification of prevalent infections as incident infections, which may inflate the estimates of cumulative incidence; (3) the possibility of not distinguishing HPV clearance from failure to detect a true HPV infection, even though two HPV negative results following one positive result were required to define HPV clearance; (4) potentially limited generalizability, as only men willing to comply with multiple clinical visits over four years were included in the study; and (5) the potential for confounding, as there may have been relevant factors related to religious or cultural practices that may also be associated with both MC status and HPV incidence or clearance that were not taken into account. 


\section{Conclusions}

Overall, our study found that circumcision status was not associated with incidence and clearance of genital HPV detection, except for among specific HPV types. However, the use in this study of a single combined sample from the penis and scrotum likely limited our ability to identify a true effect at the distal penis. Further research is needed to determine how male circumcision impacts HPV incidence and clearance by specific anatomic subsite and HPV types.

\section{Abbreviations \\ HPV: Genital human papillomavirus; MC: Male circumcision; RCT: Randomized controlled trials; HR-HPV: High-risk human papillomavirus; HIM Study: HPV Infection in men study; STIs: Sexually transmitted infections; CASI: Computer- assisted self-interview; PCR: Polymerase chain reaction; Cls: Confidence intervals; HRs: Hazard ratios; IQR: Interquartile range; aHR: Adjusted hazard ratio; RR: Rate ratio.}

\section{Competing interests}

GA: Travel grants to conferences/meetings are occasionally granted by either GlaxoSmithKline, Merck, Sanofi Pasteur MSD, Roche or Qiagen.

$G A, X C$, and FXB: My research unit is involved in HPV vaccine trials organized by GlaxoSmithKline, Merck and Sanofi Pasteur MSD.

XC: Travel grants to scientific meetings and honorarium for consultancy are occasionally granted by either GlaxoSmithKline, Merck, Sanofi Pasteur MSD.

ARG: has received research grant support from Merck \& Co., Inc. and GSK.

ARG and LLV: are on the speakers' bureau for Merck and are members of its advisory board.

AGN: has received research funding from Merck \& Co.

FXB: Travel grants to conferences/symposia/meetings and honorarium are occasionally granted by either GlaxoSmithKline, Merck, Sanofi Pasteur MSD, Roche or Qiagen.

All other authors declare that they have no competing interests.

\section{Authors' contributions}

ARG, LLV, and EL-P were the principal investigators, conceived the study, wrote the protocols, assured funding, identified clinical investigators and study personnel, and made critical revision of the manuscript. GA was responsible for conduct analysis, interpretation of data, and drafting and revision of the manuscript. WF, H-YL, and MRP made the statistical analyses, and made substantia comments to the manuscript. MA trained and supervised study clinical staff, and made substantial contributions to the manuscript. JS and MQ coordinated the field work in terms of study implementation and data collection, and made substantial comments to the manuscript. AGN, BL, XC, and FXB participated in the interpretation of data, and critical revision of the manuscript. All authors read and approved the final manuscript.

\section{Acknowledgments}

The authors would like to thank the HIM Study Teams in the USA (Moffitt Cancer Center, Tampa, FL: AR Giuliano, JL Messina, C Gage, K Eyring, K Kennedy, K Isaacs, A Bobanic, BA Sirak, MT O'Keefe, DJ Ingles, MB Schabath, CM Pierce Campbell; Johns Hopkins, Baltimore, MD: RP Viscidi), Brazil (Centro de Referência e Treinamento em DST/AIDS, Fundação Faculdade de Medicina Instituto do Câncer do Estado de São Paulo, and Ludwig Institute for Cancer Research, São Paulo: LL Villa, ML Baggio, L Galan, RJ Carvalho da Silva, L Sichero, E Gomes, E Brito, F Cernicchiaro, R Cintra, R Cunha, R Matsuo, V Souza, B Fietzek, R Hessel, V Relvas, F Silva, J Antunes, G Ribeiro, R Bocalon, R Otero, R Terreri, S Araujo, M Ishibashi, CRT-DST/AIDS nursing team), and Mexico (Instituto Mexicano del Seguro Social and Instituto Nacional de Salud Pública, Cuernavaca: E Lazcano-Ponce, A Cruz Valdez, R Alvear Vásquez, O Rojas Juárez, R González Sosa, A Salgado Morales, A Rodríguez Galván, P Román Rodríguez, A Landa Vélez, M Zepeda Mendoza, G Díaz García, V Chávez Abarca, J Ruiz Sotelo, A Gutiérrez Luna, M Hernández Nevárez, G Sánchez Martínez, A Ortiz Rojas, C Barrera Flores). The authors would also like to thank the men who provided personal information and biological samples for the study. Finally, we would like to thank Donna J. Ingles for her work copyediting the manuscript.

\section{Author details}

'Unit of Infections and Cancer (UNIC), Cancer Epidemiology Research Program (CERP), Catalan Institute of Oncology (ICO), IDIBELL. L'Hospitalet de Llobregat 08908, Barcelona, Spain. ${ }^{2}$ CIBER en Epidemiología y Salud Pública, CIBERESP (Epidemiology and Public Health Biomedical Research Consortium), Madrid, Spain. ${ }^{3}$ Program in Public Health and the Methodology of Biomedical Research, Universitat Autonoma de Barcelona (UAB). Campus Universitat Autonoma, s/n. Cerdanyola del Valles 08193, Barcelona, Spain. ${ }^{4} \mathrm{H}$. Lee Moffitt Cancer Center and Research Institute, Tampa, FL 33612, USA. ${ }^{5}$ Department of Radiology and Basic Oncology, School of Medicine, University of Sâo Paulo and School of Medical Sciences, Santa Casa de Sâo Paulo 01223-001, Brazil. ' Instituto Nacional de Salud Publica, Cuernavaca 62100, Mexico. ${ }^{7}$ Instituto Mexicano del Seguro Social, Cuernavaca 62140, Mexico. ${ }^{8}$ The University of Texas, School of Public Health, Houston, TX 77030, USA.

Received: 30 September 2013 Accepted: 5 February 2014 Published: 10 February 2014

\section{References}

1. Forman D, de Martel C, Lacey CJ, Soerjomataram I, Lortet-Tieulent J, Bruni L, Vignat J, Ferlay J, Bray F, Plummer M, Franceschi S: Global burden of human papillomavirus and related diseases. Vaccine 2012, 30(Suppl 5):F12-F23.

2. Gray RH, Serwadda D, Kong X, Makumbi F, Kigozi G, Gravitt PE, Watya S, Nalugoda F, Ssempijja V, Tobian AA, Kiwanuka N, Moulton LH, Sewankambo NK, Reynolds SJ, Quinn TC, Iga B, Laeyendecker O, Oliver AE, Wawer MJ: Male circumcision decreases acquisition and increases clearance of high-risk human papillomavirus in HIV-negative men: a randomized trial in Rakai, Uganda. J Infect Dis 2010, 201:1455-1462.

3. Lu B, Wu Y, Nielson CM, Flores R, Abrahamsen M, Papenfuss M, Harris RB, Giuliano AR: Factors associated with acquisition and clearance of human papillomavirus infection in a cohort of US men: a prospective study. $J$ Infect Dis 2009, 199:362-371.

4. Hernandez BY, Shvetsov YB, Goodman MT, Wilkens LR, Thompson P, Zhu X, Ning L: Reduced clearance of penile human papillomavirus infection in uncircumcised men. J Infect Dis 2010, 201:1340-1343.

5. Vanbuskirk K, Winer RL, Hughes JP, Feng Q, Arima Y, Lee SK, Stern ME, O'Reilly SF, Koutsky LA: Circumcision and acquisition of human papillomavirus infection in young men. Sex Transm Dis 2011, 38:1074-1081.

6. Albero G, Villa LL, Lazcano-Ponce E, Fulp W, Papenfuss MR, Nyitray AG, Lu B, Castellsague X, Abrahamsen M, Smith D, Bosch FX, Salmeron J, Quiterio M, Giuliano AR: Male circumcision and prevalence of genital human papillomavirus infection in men: a multinational study. BMC Infect Dis 2013, 13:18.

7. Giuliano AR, Lee JH, Fulp W, Villa LL, Lazcano E, Papenfuss MR, Abrahamsen M, Salmeron J, Anic GM, Rollison DE, Smith D: Incidence and clearance of genital human papillomavirus infection in men (HIM): a cohort study. Lancet 2011, 377:932-940.

8. Giuliano AR, Lazcano-Ponce E, Villa LL, Flores R, Salmeron J, Lee JH, Papenfuss MR, Abrahamsen M, Jolles E, Nielson CM, Baggio ML, Silva R, Quiterio M: The human papillomavirus infection in men study: human papillomavirus prevalence and type distribution among men residing in Brazil, Mexico, and the United States. Cancer Epidemiol Biomarkers Prev 2008, 17:2036-2043.

9. Gravitt PE, Peyton CL, Alessi TQ, Wheeler CM, Coutlee F, Hildesheim A Schiffman MH, Scott DR, Apple RJ: Improved amplification of genital human papillomaviruses. J Clin Microbiol 2000, 38:357-361.

10. Gravitt PE, Peyton CL, Apple RJ, Wheeler CM: Genotyping of 27 human papillomavirus types by using $\mathrm{L} 1$ consensus $\mathrm{PCR}$ products by a singlehybridization, reverse line blot detection method. J Clin Microbiol 1998, 36:3020-3027.

11. Bouvard V, Baan R, Straif K, Grosse Y, Secretan B, El Ghissassi F, Benbrahim-Tallaa L, Guha N, Freeman C, Galichet L, Cogliano V: A review of human carcinogens-Part B: biological agents. Lancet Oncol 2009, 10:321-322.

12. Lin DY, Wei LJ: The robust inference for the cox proportional hazards model. J Am Stat Assoc 1989, 84:1074-1078.

13. Lin DY, Wei LJ, Ying Z: Checking the Cox model with cumulative sums of martingale-based residuals. Biometrika 1993, 80:557-572.

14. Weaver BA, Feng Q, Holmes KK, Kiviat N, Lee SK, Meyer C, Stern M, Koutsky $L A$ : Evaluation of genital sites and sampling techniques for detection of human papillomavirus DNA in men. J Infect Dis 2004, 189:677-685. 
15. Nielson CM, Schiaffino MK, Dunne EF, Salemi UL, Giuliano AR: Associations between male anogenital human papillomavirus infection and circumcision by anatomic site sampled and lifetime number of female sex partners. J Infect Dis 2009, 199:7-13.

16. Hernandez BY, Wilkens LR, Zhu X, McDuffie K, Thompson P, Shvetsov YB, Ning L, Goodman MT: Circumcision and human papillomavirus infection in men: a site-specific comparison. J Infect Dis 2008, 197:787-794.

17. Tobian AA, Kong X, Gravitt PE, Eaton KP, Kigozi G, Serwadda D, Oliver AE, Nalugoda F, Makumbi F, Chen MZ, Wawer MJ, Quinn TC, Gray RH: Male circumcision and anatomic sites of penile high-risk human papillomavirus in Rakai, Uganda. Int J Cancer 2011, 129:2970-2975.

18. Partridge JM, Hughes JP, Feng Q, Winer RL, Weaver BA, Xi LF, Stern ME, Lee SK, O'Reilly SF, Hawes SE, Kiviat NB, Koutsky LA: Genital human papillomavirus infection in men: incidence and risk factors in a cohort of university students. J Infect Dis 2007, 196:1128-1136.

19. Castellsague X, Bosch FX, Munoz N, Meijer CJ, Shah KV, de Sanjose S, Eluf-Neto J, Ngelangel CA, Chichareon S, Smith JS, Herrero R, Moreno V, Franceschi S: Male circumcision, penile human papillomavirus infection, and cervical cancer in female partners. N Engl J Med 2002, 346:1105-1112.

20. Baldwin SB, Wallace DR, Papenfuss MR, Abrahamsen M, Vaught LC, Giuliano AR: Condom use and other factors affecting penile human papillomavirus detection in men attending a sexually transmitted disease clinic. Sex Transm Dis 2004, 31:601-607.

21. Auvert B, Sobngwi-Tambekou J, Cutler E, Nieuwoudt M, Lissouba P, Puren A, Taljaard D: Effect of male circumcision on the prevalence of high-risk human papillomavirus in young men: results of a randomized controlled trial conducted in Orange Farm, South Africa. J Infect Dis 2009, 199:14-19.

doi:10.1186/1471-2334-14-75

Cite this article as: Albero et al: Male circumcision and the incidence and clearance of genital human papillomavirus (HPV) infection in men: the HPV Infection in men (HIM) cohort study. BMC Infectious Diseases 2014 14:75.

\section{Submit your next manuscript to BioMed Central and take full advantage of:}

- Convenient online submission

- Thorough peer review

- No space constraints or color figure charges

- Immediate publication on acceptance

- Inclusion in PubMed, CAS, Scopus and Google Scholar

- Research which is freely available for redistribution 